\title{
Animal Models of C-Reactive Protein
}

\author{
Michael Torzewski, ${ }^{1}$ Ahmed Bilal Waqar, ${ }^{2}$ and Jianglin Fan ${ }^{2}$ \\ ${ }^{1}$ Department of Laboratory Medicine, Robert Bosch Hospital, Auerbach Street 110, 70376 Stuttgart, Germany \\ ${ }^{2}$ Department of Molecular Pathology, Interdisciplinary Graduate School of Medicine and Engineering, University of Yamanashi, \\ Yamanashi 409-3898, Japan \\ Correspondence should be addressed to Jianglin Fan; jianglin@yamanashi.ac.jp
}

Received 25 February 2014; Revised 17 March 2014; Accepted 1 April 2014; Published 24 April 2014

Academic Editor: Jan Torzewski

Copyright (c) 2014 Michael Torzewski et al. This is an open access article distributed under the Creative Commons Attribution License, which permits unrestricted use, distribution, and reproduction in any medium, provided the original work is properly cited.

As the main theme of this special issue, CRP not only is an inflammatory marker but also has diverse biological functions associated with different diseases. To investigate CRP's physiologies and their relationship with human pathological significance, it is essential to use appropriate animal models for translational research. The most popular models for the study of CRP are transgenic mice. However, researchers should be careful when extrapolating the findings derived from these animal models. This review will discuss the current concerns on CRP transgenic mice and rabbits.

\section{CRP Mouse Models}

More than 30 epidemiological studies have demonstrated a significant association between elevated serum or plasma CRP concentration and the prevalence of atherosclerotic vascular disease, the risk of recurrent cardiovascular events among those with established disease, or the incidence of first cardiovascular events among those at risk [1]. This strong base of epidemiological evidence has led to the hypothesis that CRP is both a marker of and a causal mediator for the development of atherosclerosis.

The question regarding the role of CRP in human atherogenesis is potentially clinically relevant. If CRP as a proatherogenic factor was documented, therapeutic approaches aimed at inhibiting CRP's effects in patients with atherosclerosis would obviously be of interest. Formally, experimental approaches to investigate the role of CRP in mouse models necessitated the introduction of transgenes overexpressing human or rabbit CRP to murine strains or the generation of CRP-deficient mice that have been rendered prone to atherosclerosis. The human gene, when transferred into mice, behaves as it does in man: its expression is highly inducible and tissue-specific [2]. Male CRP transgenic mice constitutively produce human CRP, with serum levels ranging between 10 and $20 \mu \mathrm{g} / \mathrm{mL}$ levels [3] that are comparable to those considered to indicate high risk in humans [4]. Furthermore, as human CRP produced endogenously in transgenic mouse completely avoids any possible contamination or other problems associated with administration of an extraneous CRP preparation, CRP transgenic mice were considered to provide an ideal model for studying the biological activities of human CRP in vivo.

Consequently, during the past ten years, a plethora of mouse studies attempted to demonstrate an atherogenic effect of CRP in genetically modified mice. Unfortunately, no clear conclusion could be drawn because these studies gave controversial and contradictory results (Table 1). Rather than answering the question of whether CRP is pro- or antiatherogenic, the following key issues and problems challenging the validity of the mouse model in general were raised as discussed.

1.1. Is CRP an Acute-Phase Protein in Mice? It is a widespread belief that, unlike human CRP, mouse CRP is not an acutephase reactant, and it is synthesized in only trace amounts [3]. However, owing to methodological shortcomings, serum levels of mouse CRP might be vastly underestimated and 


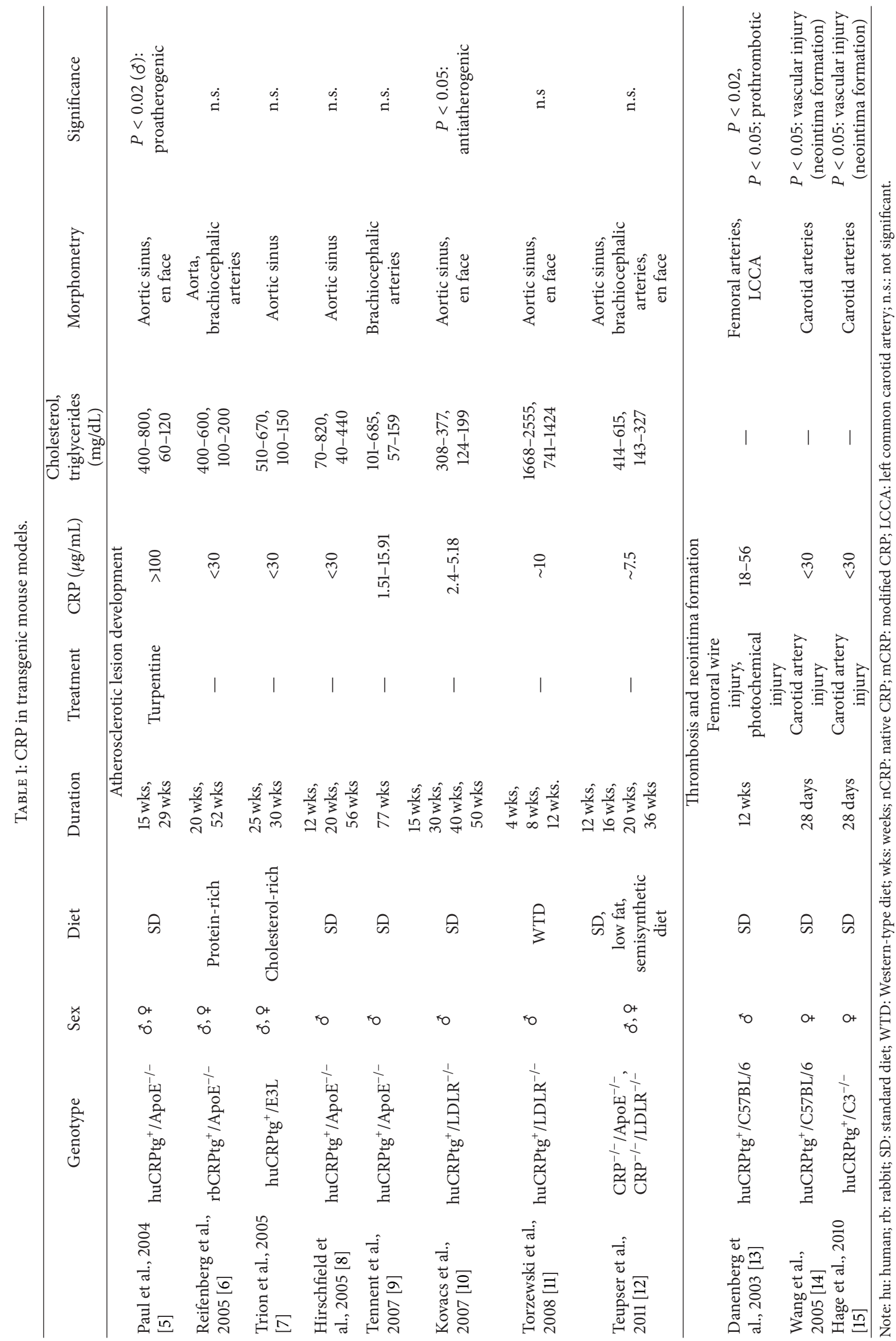


comparable to those in humans (see below) [12]. In any case, to overcome the alleged problem of insufficient CRP synthesis in mice, a transgenic mouse that overexpresses human CRP was generated, and this model is widely used to study the role of CRP in cardiovascular disease. The very first report by Paul et al. suggested that the expression of human CRP in mice accelerates aortic atherosclerotic lesion progression, thus asserting that CRP is indeed a risk factor and an active player in atherogenesis in vivo [5]. However, this interpretation has been criticised vehemently $[6,8]$, as differences in lesion size were observed only in males and only at one time point at the end of the study. Moreover, the reported difference "was of marginal statistical significance that could be abolished by elimination of a single outlier" [8], and the use of turpentine to boost circulating CRP levels periodically, which itself might induce active inflammatory pathology in the animals studied, was poorly controlled. Accordingly, the baseline and acute-phase human CRP concentrations were extraordinarily high $(100-500 \mu \mathrm{g} / \mathrm{mL})$, suggesting the presence of active intercurrent inflammatory pathology in the animals studied, for which no controls were reported. Taking these issues together, the difference in the turpentine-treated group cannot be ascribed specifically to human CRP because turpentine is a major nonspecific inflammatory stimulus.

\subsection{What Is the Gender Association of Human Transgene} CRP Expression? It was demonstrated several years ago that both constitutive and IL-6-dependent acute-phase expression of the CRP transgene in mice requires testosterone [16, 17], restricting meaningful experimental analyses of the role of CRP in cardiovascular disease to male CRP transgenic mice. Surprisingly, despite the widespread knowledge that the expression of transgenic human CRP is under strict testosterone control, female mice were repeatedly included in the respective animal studies $[5,7]$. It is no wonder that female mice were not those that provided positive results if any were obtained.

1.3. Which Atherosclerosis-Prone Mouse Model Mimics Human-Like Hypercholesterolemia? Knockout mice with a defect in either apolipoprotein $\mathrm{E}\left(\mathrm{ApoE}^{-/-}\right)$[18] or low-densitylipoprotein receptor $\left(\mathrm{LDLR}^{-/-}\right.$) [19] develop atherosclerotic lesions and are currently widely used as models for investigating the pathomechanisms underlying atherosclerosis. The conflicting results obtained partly suggest that the effects of CRP on atherosclerosis are dependent on the mouse model used. First of all, ApoE ${ }^{-/-}$mice have far more severe hypercholesterolemia than humans, and most of their cholesterol is contained in very low-density lipoproteins (VLDL) rather than in low-density lipoproteins (LDLs) as in humans. This issue is of high relevance as the effects of CRP on lesion development may be influenced by differences in the degree and type of hyperlipoproteinemia in the mouse models and the apoE protein per se can alter immune responses [20]. Immune responses that may directly involve apoE include phagocytosis of apoptotic bodies, altered macrophage dynamics [21] and altered antigen presentation efficiency [22]. Thus, the effects of human CRP on mouse atherosclerotic lesion development may be masked in $\mathrm{ApoE}^{-/-}$mice because they are VLDL animals and have altered immune functions.

Consequently, the $\mathrm{ApoE}^{-/-}$mouse model may not be ideal for studies of human CRP in atherosclerosis. In contrast, the $\mathrm{LDLR}^{-/-}$model may be superior for a number of reasons. First, atherosclerotic lesions do not develop spontaneously as in $\mathrm{ApoE}^{-/-}$animals but are inducible under a Westerntype diet (WTD). Second, the serum lipoprotein pattern of $\mathrm{LDLR}^{-/-}$animals is characterized by high-level LDL rather than chylomicrons and VLDL (as in $\mathrm{ApoE}^{-/-}$mice) and thus more closely mimics the situation in humans $[6,10,11]$.

1.4. What Is the Functional Role of Human CRP as a Foreign Protein in Mouse? It is unsurprising that there are no experimental animal models of CRP function that completely replicate the human situation. Human CRP is a foreign protein in the mouse, with many uncertainties concerning its functional role in the immune system of these animals. The situation becomes even more complicated when these mice are crossed with ApoE-deficient mice that lack a fully functional complement system. The study by Reifenberg et al. uncovered the disturbing facts that the interactions among CRP, complement, and LDL, as have been delineated in humans, may not exist similarly in mice [6]. It cannot be ruled out that mouse CRP might be active, but the inability of transgenic CRP to execute one of its primary functions (complement activation) places obvious constraints on the validity of this animal model. Moreover, transgenic human CRP operating in a xenogeneic murine environment might also fail to interact with further important mouse effector molecules, such as cellular receptors, the extracellular matrix, and lipoproteins, as pointed out by a number of studies $[6,10,12,23]$. Thus, it is difficult to determine which of the reports are valid, because the model itself encounters several problems.

To help to overcome the above-mentioned issues, Reifenberg et al. crossbred mice expressing rabbit CRP (rbCRP) onto apoE knockout animals and studied the effect on atherogenesis [6]. Expression of the rbCRP transgene is independent of gender, and an additional inflammatory stimulus is also not required. rbCRP and human CRP are similar in structure and function. Both bind phosphocholine, C-polysaccharide, polycations, chromatin, and histones, activate complement and protect mice from lethal challenges with pneumococci [24-30]. However, no marked effect on the formation of moderately advanced atherosclerotic lesions could be discerned, either in male or in female apoE knockout mice.

1.5. What about the CRP-Deficient Mouse? Owing to doubts about the physiological role of genuine mouse CRP, overexpression rather than deletion of CRP was regarded as the only meaningful way to investigate the impact of CRP on murine atherogenesis. As already mentioned above, however, serum levels of mouse CRP might be vastly underestimated and comparable to those of humans, challenging the 
dogma of insufficient CRP synthesis in mice. Recently, a complementary approach was chosen, generating mice with targeted deletion of the CRP gene on B6.ApoE ${ }^{-/-}$as well as B6.LDLR ${ }^{-/}$genetic backgrounds [12]. This approach avoids the above-mentioned xenogeneic complications of CRP overexpression. Reliable commercial reagents along with serum CRP knockout mice as a stringent negative control indicated sufficient expression of mouse CRP even in the noninduced state. On the basis of quantitative analysis of atherosclerotic lesions, the data suggested that mouse CRP may even mediate atheroprotective effects rather than having a proatherogenic role in the two most widely used mouse models of atherosclerosis. These results together with the results by Torzewski [11] add a cautionary note to the idea of targeting CRP as therapeutic intervention against progressive cardiovascular disease and point out that CRP might actually serve a physiological and primarily nonharmful function, as first proposed in 2004 [31].

1.6. Is There an Association of CRP with Atherothrombosis rather than Just Atheroma? One has to bear in mind that most of the epidemiological studies demonstrating a significant association between elevated serum or plasma CRP concentration and the prevalence of human atherosclerotic vascular disease refer not to atherosclerotic lesion development but rather to its clinical sequelae caused by plaque rupture and thrombosis. It is therefore justified to ask whether there is an association of CRP with atherothrombosis rather than just atheroma in animal models. The respective studies, however, are far from being as numerous as those on atherosclerotic lesion development. A first report showed that occlusive thrombosis was more pronounced in CRP transgenic mice than in wild-type mice [13]. More recently, it was demonstrated that there is a more exaggerated response to vascular injury in human CRP transgenic mice [14] and that this response requires complement [15]. Caution on the interpretation of these data is warranted, however, because the vascular remodeling process associated with carotid artery ligation versus atherogenesis is not the same.

1.7. Conclusions. In conclusion, it is evident that each one of the above-mentioned genetically engineered mouse models addresses some of the discussed key issues and problems but leaves enough unresolved problems to call the respective mouse model into question. This can be extended to any "key issue" in atherosclerosis research that mouse models have claimed to address. They have led to very little true advance and, yet more importantly, they have generated many false and confusing concepts. Thus, caution should be exercised when extrapolating observations in genetically engineered mouse models with incompletely characterized physiological alterations to the situation in human disease. Finally, it may be appropriate to say that it was worth generating these mouse model systems, but they hardly enable us to answer definitively whether or not CRP actively contributes to human atherogenesis.
TABLE 2: Comparison of CRP in different species.

\begin{tabular}{|c|c|c|c|}
\hline & Mouse CRP & Rabbit CRP & Human CRP \\
\hline M.W. (Kd) & 19.5 & 22 & 21 \\
\hline Plasma levels & $<2 \mathrm{mg} / \mathrm{L}$ & $\begin{array}{c}<3 \mathrm{mg} / \mathrm{L} \\
>100 \mathrm{mg} / \mathrm{L}^{*}\end{array}$ & $\begin{array}{c}<1 \mathrm{mg} / \mathrm{L} \\
>10,000 \mathrm{mg} / \mathrm{L}^{*}\end{array}$ \\
\hline $\begin{array}{l}\text { Activation of } \\
\text { complement }\end{array}$ & No & Yes & Yes \\
\hline $\begin{array}{l}\text { Binding to plasma } \\
\text { LDLs }\end{array}$ & ** & Yes & Yes \\
\hline $\begin{array}{l}\text { Deposition in the } \\
\text { lesions of } \\
\text { atherosclerosis }\end{array}$ & No & Yes & Yes \\
\hline
\end{tabular}

${ }^{*}$ In acute inflammatory state.

** Wild-type mice do not have LDLs as in humans and rabbits.

Also see [6, 32-38] for details.

\section{Rabbits as an Alternative CRP Model for Studying Human CRP}

As described above, there are many problems of using mouse models for the study of CRP's physiological functions and controversies have risen in regard to CRP's roles in atherosclerosis. To overcome these problems, alternative animals are needed; here, we focus on using rabbit models as another means of investigating CRP biology.

2.1. Rabbit CRP versus Mouse CRP: Which One Is Closest to Human CRP? The molecular and physiological features of rabbit CRP more closely resemble those of human CRP compared with mouse CRP in several aspects (Table 2) [32, 33]. First, like human CRP but unlike mouse CRP, rabbit CRP acts as a major acute-phase reactant (inflammatory marker) in the plasma and thus CRP levels are increased up to $\sim 100 \mathrm{mg} / \mathrm{L}$ upon inflammatory stimulation [34]. It is well known that, in mouse plasma, the major inflammatory marker is serum amyloid protein (SAP) rather than CRP; plasma CRP levels in mouse are normally markedly lower than those in rabbits and humans and do not fluctuate regardless of the presence of inflammation [35]. Secondly, rabbit CRP can strongly bind with plasma atherogenic lipoproteins [36], like human CRP [37]. Thirdly, CRP immunoreactive proteins are present in all types of lesion of both rabbit and human atherosclerosis [38], but no CRP was detected in the lesions of mouse. These features of rabbit CRP lead to the notion that rabbits may be an ideal model (or a better model than mouse) for examining the physiological and pathophysiological roles of human CRP [23].

2.2. Rabbit Atherosclerosis Models for CRP. Rabbits have been used as an excellent model for the study of human atherosclerosis because their lipoprotein metabolism and cardiovascular system are similar to those of humans [39]. Unlike mice, but like humans, rabbits have abundant plasma cholesteryl ester transfer protein, an important regulator of cholesterol transfer, and exhibit hepatic apoB100 and intestinal apoB48 synthesis, and their lipoprotein profiles are rich in low-density lipoproteins (LDL) whereas mice are deficient 
in cholesteryl ester transfer protein and their plasma lipoproteins are dominated by high-density lipoproteins (HDL). Rabbits are sensitive to a cholesterol-rich diet and develop atherosclerosis rapidly, whereas most strains of wild-type mice are resistant to a cholesterol-induced atherosclerosis. WHHL rabbits provide another means of studying human familial hypercholesterolemia and atherosclerosis because these rabbits are deficient in LDL receptors [40]. A decade ago, we used both cholesterol-fed rabbits and WHHL rabbits and revealed several important features of CRP and their relationship with atherosclerosis [38]. We first found that plasma CRP levels are increased in hypercholesterolemic rabbits and correlated with the severity of aortic lesion size. Secondly, we found that CRP immunoreactive proteins are present in the lesions of atherosclerosis of rabbits regardless of the lesion types. Basically, CRP is associated mainly with extracellular matrix and seldom with macrophages. CRP is also closely colocalized with apoB and the terminal complement complex, suggesting that possible interactions between CRP-apoB-complement are present in the lesions $[31,38,41]$. Thirdly, CRP is basically synthesized by the liver rather than the vascular wall (such as macrophages). The consensus is that it is hepatically synthesized CRP that regulates plasma levels of CRP [42]. Despite this, the presence of CRP deposition in the lesions of atherosclerosis sustained efforts in the cardiovascular field during the last decade to elucidate whether CRP truly constitutes another risk factor for alongside hypercholesterolemia and is indeed involved in the initiation and progression of atherosclerotic disease. If CRP is proinflammatory and atherogenic, can we target CRP for the prevention and treatment of atherosclerosis [23]? These findings obtained from rabbit studies further strengthened the notion that rabbits are an excellent model for illustrating the relationship between CRP and atherosclerosis. Nevertheless, this study using hypercholesterolemic rabbits still cannot answer the question of whether CRP is a mediator or a marker of atherosclerosis [43].

2.3. Human CRP Transgenic Rabbit Model. To elucidate whether high levels of plasma CRP participate in the development of atherosclerosis, our laboratory generated 2 lines of transgenic ( $\mathrm{Tg}$ ) rabbits expressing human CRP (hCRP) transgene in the liver [44]. Plasma levels of hCRP were $0.4 \pm$ $0.13 \mathrm{mg} / \mathrm{L}$ and $57.8 \pm 20.6 \mathrm{mg} / \mathrm{L}$ in these two lines of $\mathrm{Tg}$ rabbits, respectively. The expression of hCRP does not cause any health disorders or phenotypes (such as spontaneous atherosclerosis) in Tg rabbits. hCRP isolated from Tg rabbit plasma exhibited the ability to activate rabbit complement, suggesting that human CRP is indeed functional in Tg rabbits [44]. Using this powerful model, we compared the susceptibility of Tg rabbits to cholesterol-rich diet-induced aortic and coronary atherosclerosis with that of non-Tg rabbits. To our surprise, neither high nor low plasma concentrations of hCRP affected aortic or coronary atherosclerotic lesion formation in Tg rabbits, even though a massive amount of hCRP was detected in the lesions of atherosclerosis [44]. Therefore, high levels of plasma and lesional CRP in Tg rabbits do not enhance the development of atherosclerosis.
While these results are disappointing to CRP believer, this study cannot exclude the possibility that CRP participates in other pathological processes such as thrombosis, myocardial infarction, and arthritis. On these issues, we performed a series of experiments using hCRP Tg rabbits.

Using double balloon-injury models of the femoral arteries in $\mathrm{Tg}$ rabbits, we demonstrated that high expression of hCRP led to enhanced thrombosis formation on the injured smooth muscle cell-rich neointima by upregulating tissue factor expression [45], suggesting that CRP mediates thrombosis. In addition to atherosclerosis, CRP along with complement activation has been shown to accelerate myocardial infarction in rats and targeting CRP can prevent CRPinduced myocardial injury $[46,47]$. However, this hypothesis has not been tested in other models, including both mouse and rabbit. Using the coronary ligation method, we generated acute myocardial infarction models in Tg rabbits. In preliminary experiments, we did not find any significant roles of CRP on myocardial infarction size or plasma cardiac markers (Waqar et al. unpublished data).

\subsection{Effects of CRP Antisense Oligonucleotides on WHHL} Rabbits. The major concern about using cholesterol-fed Tg animals is that transgenic proteins (namely, human CRP) are exogenous to animals and the atherogenic lipoproteins are remnant lipoproteins, so-called $\beta$-VLDLs, which may complicate the evaluation of hCRP pathophysiological functions in these models [44]. To overcome this drawback, we attempted to use a therapeutic approach to inhibit endogenous CRP and then examined the CRP-lowering effect. Towards this goal, in collaboration with ISIS Pharmaceuticals, Inc., we designed and injected robust rabbit CRP antisense oligonucleotides into WHHL rabbits, which have elevated plasma CRP levels (10 20-fold higher than in wildtype rabbits) in addition to having atherosclerosis. While tremendous efforts were made in this regard, we failed to demonstrate any therapeutic effects on atherosclerosis in WHHL rabbits (see accompanied paper by $\mathrm{Yu}$ et al. in this special issue).

2.5. Conclusions. In conclusion, rabbits are a suitable model for the investigation of CRP physiology because they resemble humans in many aspects, compared with mice. Cholesterol-fed rabbits along with WHHL rabbits and hCRP transgenic rabbits offer another opportunity to elucidate CRP functions that cannot be conducted in mice. After a decade's effort using these unique models, it is time to draw a conclusion regarding the true role of CRP in atherosclerosis. Is CRP a marker or mediator of atherosclerosis? Do we still need to continue the debate? Should we treat CVD patients who have a high level of CRP? The answers are becoming clearer and clearer: without doubt, plasma CRP levels are indeed increased and CRP is intimately present in the lesions of atherosclerosis. However, the net effect exerted by CRP is not proatherogenic, while we cannot rule out the possibility that CRP is antiatherogenic or participates in other diseases. These experimental observations are also in support of the human studies reported recently [48-51]. These studies also 
told us that it is unlikely that CRP can be a therapeutic target for the prevention and treatment of cardiovascular diseases.

\section{Conflict of Interests}

The authors declare that there is no conflict of interests regarding the publication of this paper.

\section{References}

[1] S. A. Halim, L. K. Newby, and E. M. Ohman, "Biomarkers in cardiovascular clinical trials: past, present, future," Clinical Chemistry, vol. 58, no. 1, pp. 45-53, 2012.

[2] G. Ciliberto, R. Arcone, E. F. Wagner, and U. Rüther, "Inducible and tissue-specific expression of human C-reactive protein in transgenic mice," EMBO Journal, vol. 6, no. 13, pp. 4017-4022, 1987.

[3] A. J. Szalai and M. A. McCrory, "Varied biologic functions of C-reactive protein: lessons learned from transgenic mice," Immunologic Research, vol. 26, no. 1-3, pp. 279-287, 2002.

[4] P. M. Ridker, M. Cushman, M. J. Stampfer, R. P. Tracy, and C. H. Hennekens, "Inflammation, aspirin, and the risk of cardiovascular disease in apparently healthy men," The New England Journal of Medicine, vol. 336, no. 14, pp. 973-979, 1997.

[5] A. Paul, K. W. Ko, L. Li et al., "reactive protein accelerates the progression of atherosclerosis in apolipoprotein E-deficient mice," Circulation, vol. 109, no. 5, pp. 647-655, 2004.

[6] K. Reifenberg, H.-A. Lehr, D. Baskai et al., "Role of C-reactive protein in atherogenesis: can the apolipoprotein E knockout mouse provide the answer?" Arteriosclerosis, Thrombosis, and Vascular Biology, vol. 25, no. 8, pp. 1641-1646, 2005.

[7] A. Trion, M. P. M. de Maat, J. W. Jukema et al., "No effect of C-reactive protein on early atherosclerosis development in apolipoprotein $\mathrm{E}^{*} 3$-Leiden/human C-reactive protein transgenic mice," Arteriosclerosis, Thrombosis, and Vascular Biology, vol. 25, no. 8, pp. 1635-1640, 2005.

[8] G. M. Hirschfield, J. R. Gallimore, M. C. Kahan et al., "Transgenic human C-reactive protein is not proatherogenic in apolipoprotein E-deficient mice," Proceedings of the National Academy of Sciences of the United States of America, vol. 102, no. 23, pp. 8309-8314, 2005.

[9] G. A. Tennent, W. L. Hutchinson, M. C. Kahan et al., "Transgenic human CRP is not pro-atherogenic, proatherothrombotic or pro-inflammatory in apoE-/- mice," Atherosclerosis, vol. 196, pp. 248-255, 2007.

[10] A. Kovacs, P. Tornvall, R. Nilsson, J. Tegnér, A. Hamsten, and J. Björkegren, "Human C-reactive protein slows atherosclerosis development in a mouse model with human-like hypercholesterolemia," Proceedings of the National Academy of Sciences of the United States of America, vol. 104, no. 34, pp. 13768-13773, 2007.

[11] M. Torzewski, K. Reifenberg, F. Cheng et al., "No effect of C-reactive protein in LDLR-/- / human C-reactive protein transgenic mice," Thrombosis and Haemostasis, vol. 99, pp. 196201, 2008.

[12] D. Teupser, O. Weber, T. N. Rao, K. Sass, J. Thiery, and H. Jörg Fehling, "No reduction of atherosclerosis in C-reactive protein (CRP)-deficient mice," Journal of Biological Chemistry, vol. 286, no. 8, pp. 6272-6279, 2011.

[13] H. D. Danenberg, A. J. Szalai, R. V. Swaminathan et al., "Increased thrombosis after arterial injury in human C-reactive protein-transgenic mice," Circulation, vol. 108, no. 5, pp. 512515, 2003.

[14] D. Wang, S. Oparil, Y. F. Chen et al., "Estrogen treatment abrogates neointima formation in human C-reactive protein transgenic mice," Arteriosclerosis, Thrombosis, and Vascular Biology, vol. 25, no. 10, pp. 2094-2099, 2005.

[15] F. G. Hage, S. Oparil, D. Xing, Y. F. Chen, M. A. McCrory, and A. J. Szalai, "C-reactive protein-mediated vascular injury requires complement," Arteriosclerosis, Thrombosis, and Vascular Biology, vol. 30, no. 6, pp. 1189-1195, 2010.

[16] A. J. Szalai, D. E. Briles, and J. E. Volanakis, "Human C-reactive protein is protective against fatal Streptococcus pneumoniae infection in transgenic mice," Journal of Immunology, vol. 155, no. 5, pp. 2557-2563, 1995.

[17] A. J. Szalai, F. W. van Ginkel, S. A. Dalrymple, R. Murray, J. R. McGhee, and J. E. Volanakis, "Testosterone and IL-6 requirements for human $\mathrm{C}$-reactive protein gene expression in transgenic mice," Journal of Immunology, vol. 160, no. 11, pp. 5294-5299, 1998.

[18] J. A. Piedrahita, S. H. Zhang, J. R. Hagaman, P. M. Oliver, and N. Maeda, "Generation of mice carrying a mutant apolipoprotein E gene inactivated by gene targeting in embryonic stem cells," Proceedings of the National Academy of Sciences of the United States of America, vol. 89, no. 10, pp. 4471-4475, 1992.

[19] S. Ishibashi, M. S. Brown, J. L. Goldstein, R. D. Gerard, R. E. Hammer, and J. Herz, "Hypercholesterolemia in low density lipoprotein receptor knockout mice and its reversal by adenovirus-mediated gene delivery," Journal of Clinical Investigation, vol. 92, no. 2, pp. 883-893, 1993.

[20] D. T. Laskowitz, D. M. Lee, D. Schmechel, and H. F. Staats, "Altered immune responses in apolipoprotein E-deficient mice," Journal of Lipid Research, vol. 41, no. 4, pp. 613-620, 2000.

[21] D. J. Grainger, J. Reckless, and E. McKilligin, "Apolipoprotein E modulates clearance of apoptotic bodies in vitro and in vivo, resulting in a systemic proinflammatory state in apolipoprotein E-deficient mice," Journal of Immunology, vol. 173, no. 10, pp. 6366-6375, 2004.

[22] C. Tenger and X. Zhou, "Apolipoprotein E modulates immune activation by acting on the antigen-presenting cell," Immunology, vol. 109, no. 3, pp. 392-397, 2003.

[23] J. Torzewski, "C-reactive protein and atherogenesis: new insights from established animal models," The American Journal of Pathology, vol. 167, no. 4, pp. 923-925, 2005.

[24] B. A. Bach, H. Gewurz, and A. P. Osmand, " $\mathrm{C}$ reactive protein in the rabbit: Isolation, characterization and binding affinity to phosphocholine," Molecular Immunology, vol. 14, no. 3, pp. 215219, 1977.

[25] S. Black, A. Agrawal, and D. Samols, "The phosphocholine and the polycation-binding sites on rabbit C-reactive protein are structurally and functionally distinct," Molecular Immunology, vol. 39, no. 16, pp. 1045-1054, 2003.

[26] T. J. Dougherty, H. Gewurz, and J. N. Siegel, "Preferential binding and aggregation of rabbit c-reactive protein with arginine-rich proteins," Molecular Immunology, vol. 28, no. 10, pp. 1113-1120, 1991.

[27] J. Horowitz, J. E. Volanakis, and D. E. Briles, "Blood clearance of Streptococcus pneumoniae by C-reactive protein," Journal of Immunology, vol. 138, no. 8, pp. 2598-2603, 1987.

[28] C. S. Lin, D. Xia, J. S. Yun et al., "Expression of rabbit C-reactive protein in transgenic mice," Immunology and Cell Biology, vol. 73, no. 6, pp. 521-531, 1995. 
[29] E. B. Oliveira, E. C. Gotschlich, and T. Y. Liu, "Comparative studies on the binding properties of human and rabbit Creactive proteins," Journal of Immunology, vol. 124, no. 3, pp. 1396-1402, 1980.

[30] F. A. Robey, K. D. Jones, T. Tanaka, and T. Y. Liu, "Binding of C-reactive protein to chromatin and nucleosome core particles. A possible physiological role of C-reactive protein," Journal of Biological Chemistry, vol. 259, no. 11, pp. 7311-7316, 1984.

[31] S. Bhakdi, M. Torzewski, K. Paprotka et al., "Possible protective role for $\mathrm{C}$-reactive protein in atherogenesis: complement activation by modified lipoproteins halts before detrimental terminal sequence," Circulation, vol. 109, no. 15, pp. 1870-1876, 2004.

[32] I. Kushner and G. Feldmann, "Control of the acute phase response. Demonstration of C-reactive protein synthesis and secretion by hepatocytes during acute inflammation in the rabbit," Journal of Experimental Medicine, vol. 148, no. 2, pp. 466-477, 1978.

[33] H. C. Anderson and M. McCarty, "The occurrence in the rabbit of an acute phase protein analogous to human $\mathrm{C}$ reactive protein," The Journal of experimental medicine, vol. 93, no. 1, pp. 25-36, 1951.

[34] M. B. Pepys and M. L. Baltz, "Acute phase proteins with special reference to C-reactive protein and related proteins (pentaxins) and serum amyloid A protein," Advances in Immunology, vol. 34, pp. 141-212, 1983.

[35] M. B. Pepys, M. Baltz, and K. Gomer, "Serum amyloid Pcomponent is an acute-phase reactant in the mouse," Nature, vol. 278, no. 5701, pp. 259-261, 1979.

[36] I. F. Rowe, A. K. Soutar, and I. M. Trayner, "Rabbit and rat C-reactive proteins bind apolipoprotein B-containing lipoproteins," Journal of Experimental Medicine, vol. 159, no. 2, pp. 604616, 1984.

[37] F. C. de Beer, A. K. Soutar, and M. L. Baltz, "Low density lipoprotein and very low density lipoprotein are selectively bound by aggregated C-reactive protein," Journal of Experimental Medicine, vol. 156, no. 1, pp. 230-242, 1982.

[38] H. Sun, T. Koike, T. Ichikawa et al., "C-reactive protein in atherosclerotic lesions: Its origin and pathophysiological significance," American Journal of Pathology, vol. 167, no. 4, pp. 11391148, 2005.

[39] J. Fan and T. Watanabe, "Transgenic rabbits as therapeutic protein bioreactors and human disease models," Pharmacology and Therapeutics, vol. 99, no. 3, pp. 261-282, 2003.

[40] M. Shiomi and T. Ito, "The Watanabe heritable hyperlipidemic (WHHL) rabbit, its characteristics and history of development: a tribute to the late Dr. Yoshio Watanabe," Atherosclerosis, vol. 207, no. 1, pp. 1-7, 2009.

[41] J. Torzewski, M. Torzewski, D. E. Bowyer et al., "C-Reactive protein frequently colocalizes with the terminal complement complex in the intima of early atherosclerotic lesions of human coronary arteries," Arteriosclerosis, Thrombosis, and Vascular Biology, vol. 18, no. 9, pp. 1386-1392, 1998.

[42] I. Jialal, S. Devaraj, U. Singh, J. Fan, and E. Chen, "Sources of CRP in atherosclerotic lesions," The American Journal of Pathology, vol. 168, no. 3, pp. 1055-1056, 2006.

[43] I. Jialal, S. Devaraj, U. Singh, J. Fan, and E. Chen, "Sources of CRP in atherosclerotic lesions," The American Journal of Pathology, vol. 168, no. 3, pp. 1054-1056, 2006.

[44] T. Koike, S. Kitajima, Y. Yu et al., "Human C-reactive protein does not promote atherosclerosis in transgenic rabbits," Circulation, vol. 120, no. 21, pp. 2088-2094, 2009.
[45] S. Matsuda, A. Yamashita, Y. Sato et al., "Human C-reactive protein enhances thrombus formation after neointimal balloon injury in transgenic rabbits," Journal of Thrombosis and Haemostasis, vol. 9, no. 1, pp. 201-208, 2011.

[46] M. Griselli, J. Herbert, W. L. Hutchinson et al., "C-reactive protein and complement are important mediators of tissue damage in acute myocardial infarction," Journal of Experimental Medicine, vol. 190, no. 12, pp. 1733-1740, 1999.

[47] M. B. Pepys, G. M. Hirschfield, G. A. Tennent et al., "Targeting C-reactive protein for the treatment of cardiovascular disease," Nature, vol. 440, no. 7088, pp. 1217-1221, 2006.

[48] S. Kaptoge, E. di Angelantonio, G. Lowe et al., "C-reactive protein concentration and risk of coronary heart disease, stroke, and mortality: an individual participant meta-analysis," The Lancet, vol. 375, pp. 132-140, 2010.

[49] F. Wensley, P. Gao, S. Burgess et al., "Association between $\mathrm{C}$ reactive protein and coronary heart disease: mendelian randomisation analysis based on individual participant data," British Medical Journal, vol. 342, article d548, 2011.

[50] S. Kaptoge, E. di Angelantonio, L. Pennells et al., "C-reactive protein, fibrinogen, and cardiovascular disease prediction," The New England Journal of Medicine, vol. 367, pp. 1310-1320, 2012.

[51] T. Lane, N. Wassef, S. Poole et al., "Infusion of pharmaceuticalgrade natural human C-reactive protein is not proinflammatory in healthy adult human volunteers," Circulation Research, vol. 114, pp. 672-676, 2014. 


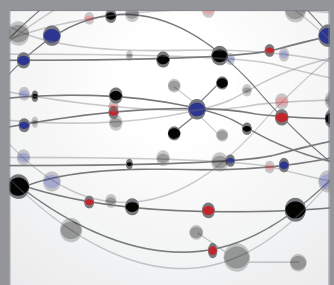

The Scientific World Journal
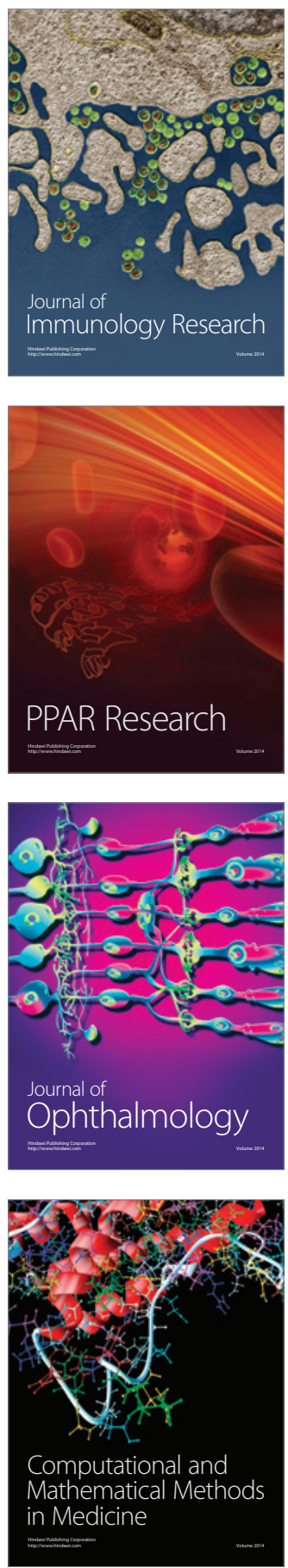

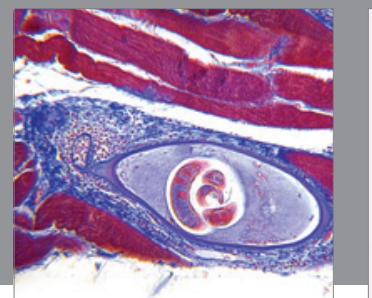

Gastroenterology

Research and Practice
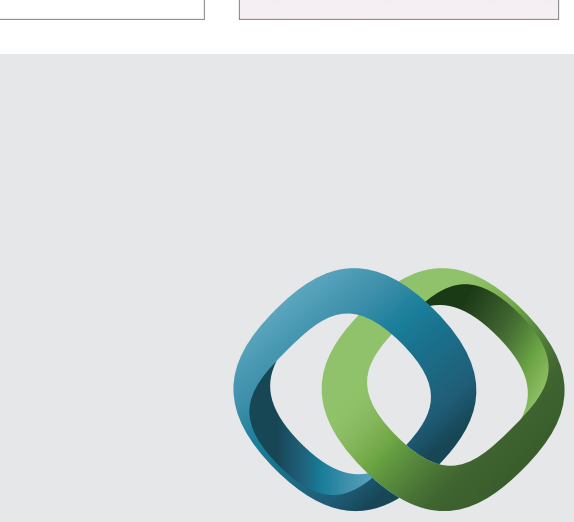

\section{Hindawi}

Submit your manuscripts at

http://www.hindawi.com
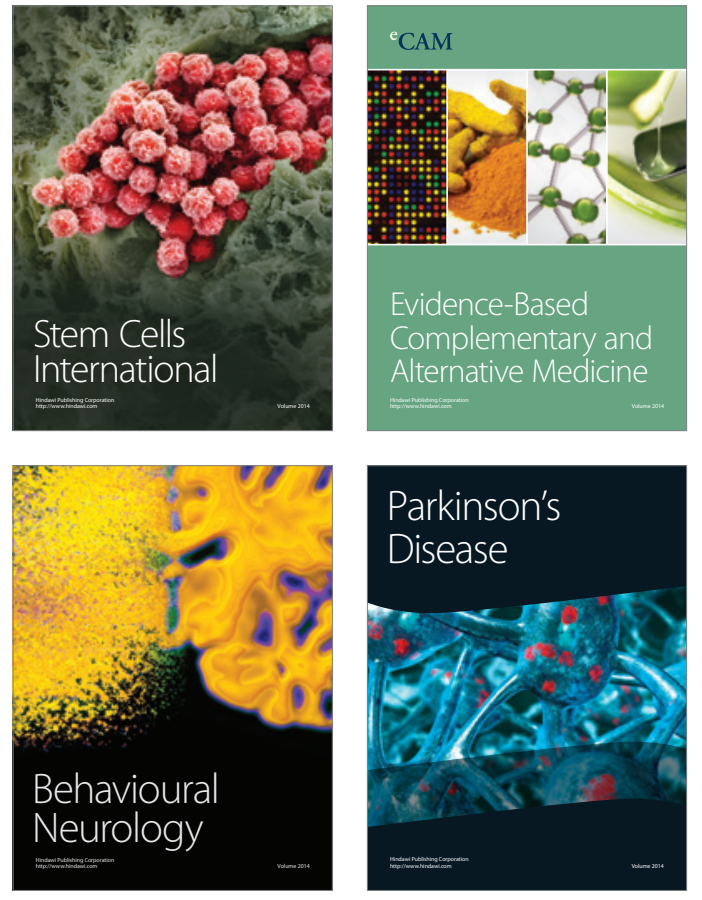
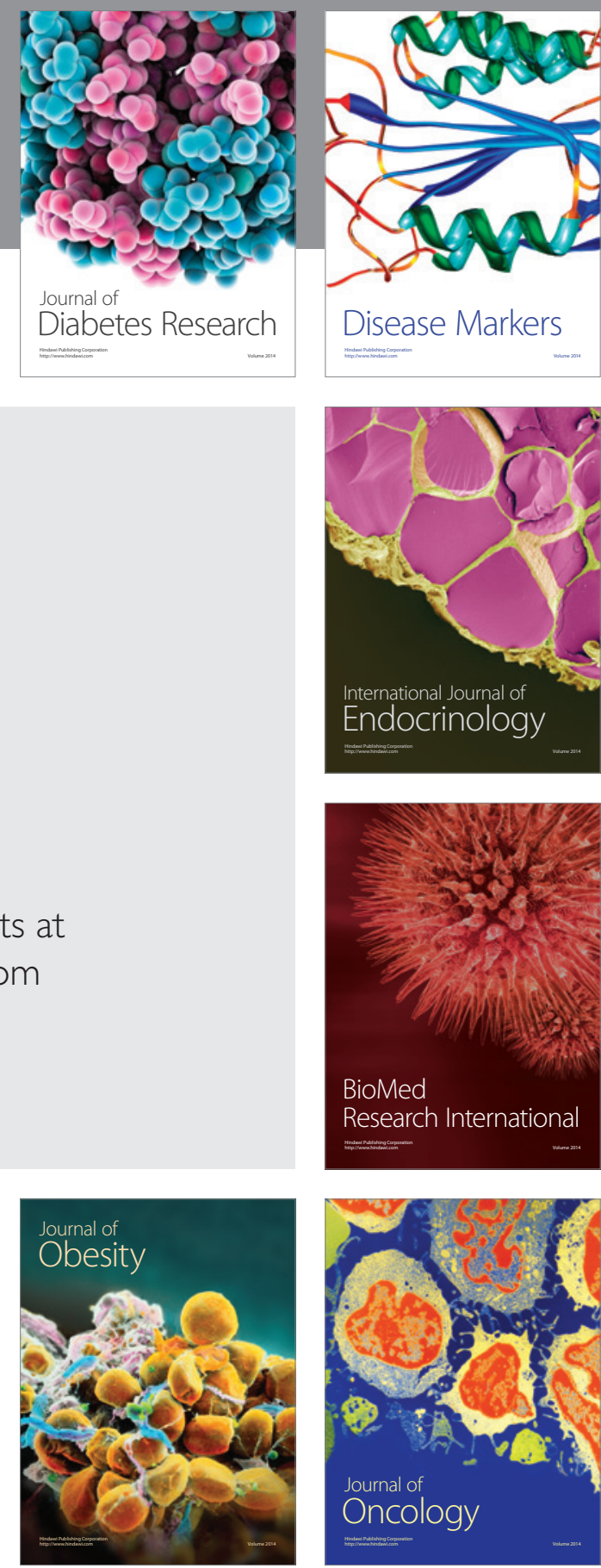

Disease Markers
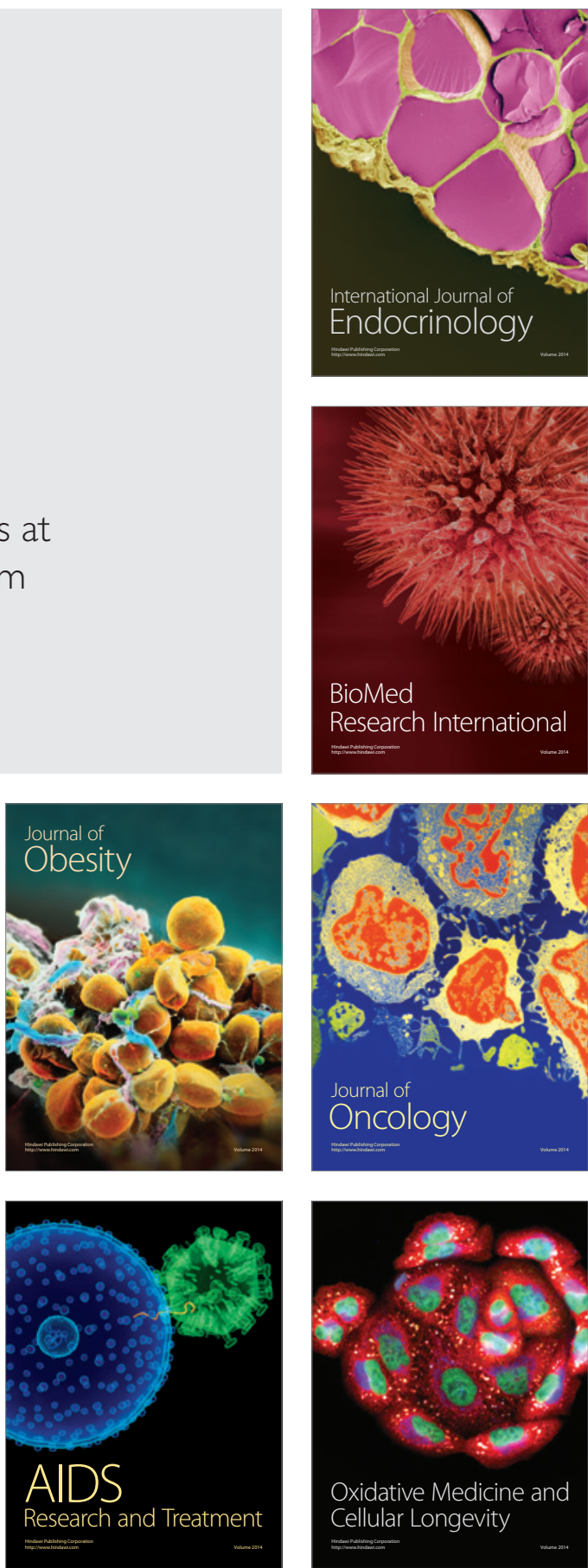